\title{
Performance Mapping of an Aluminum - Nitinol Composite Vibrating Beam
}

\author{
Nicholas G. Garafolo*, Rachel J. Collard \\ Department of Mechanical Engineering, The University of Akron, Akron, OH 44325, U.S.A. \\ *Corresponding author: nicholas.g.garafolo@uakron.edu
}

\begin{abstract}
Dynamic control of a vibrating beam is critical to the efforts of mitigation of high cycle fatigue, as it is a leading cause of component or engine failure. Recent advances in composite structures have afford the ability to control the eigenvalue, eigenvectors, and amplitude of vibration through the use of the shape memory effect of shape memory alloys (e.g. Nitinol). These "smart materials" are proven to create active damping and variable stiffness in engine components is an innovative concept. Research presented herein seeks to quantify the effectiveness of Nitinol as a HCF mitigation technique and map the frequency and damping performance. A composite beam consisting of a Nitinol topical actuator adhered to an aluminum alloy 6061 substrate was designed and fabricated. Test specimens comprised two configurations: (1) a composite beam with the topical treatment encompassing the full span of free length of the beam, and (2) a composite beam with the topical treatment encompassing half the span the free length of the beam. Benchtop tests allowed for the determination both modal frequency and damping of the aluminum Nitinol composite beam with an 8 in. free length. Modal analyses were taken over a selected frequency range using a single point laser vibrometer, excited using a dynamic shaker. Experimental studies were completed over a frequency range which represented the second bending mode. Quality factor values, Q, of approximately 200 were observed. No correlation, however, with phase transformation was realized. The design space charts for temperature, modal frequency, and beam tip amplitude were compiled for both the full-span and half-span sample for second bending mode. These charts graphically depict how tip amplitude changes with varied temperature and illustrate the design capabilities created through the use of shape memory alloy components in dynamic applications.
\end{abstract}

Keywords: shape memory alloys, thin foil, vibration, high cycle fatigue, turbomachinery, damping

Cite This Article: Nicholas G. Garafolo, and Rachel J. Collard, "Performance Mapping of an Aluminum Nitinol Composite Vibrating Beam." Journal of Mechanical Design and Vibration, vol. 5, no. 1 (2017): 27-36. doi: $10.12691 / \mathrm{jmdv}-5-1-4$.

\section{Introduction}

High cycle fatigue (HCF) mitigation requires research into novel materials and techniques to expand the available tools and resources for innovated designs. Recent advances in the use of Shape Memory Alloys (SMA) for controlling the vibration characteristics offer significant potential for HCF mitigation cite [1]. High cycle fatigue, as characterized by high frequencies, low amplitudes, cyclic elastic behavior, and high cycle count, affect a large number of engine components. The root causes include unidentified resonance, manufacturing problems, foreign object damage, and aero-elasticity issues [2].

The balance of aerodynamics performance and damping is difficult, as the means to obtain additional damping cannot interfere with the aerodynamics of machinery blades, so any treatments must be internal, out of air flow, or in the form of a thin layer. Emerging materials and unique methods for their implementation are vital for the success of novel HCF mitigation methods.

Current mitigation techniques include sprayed coatings, constrainment layers, and piezo-electric actuators [3]. Coatings, including magnesium aluminate spinel, have been shown to increase damping nonlinearly in titanium blades [4,5]. Active vibration control using piezo-electric actuators have gained acceptance because they have no moving parts, they are low weight, and they provide high force with low power consumption. Piezo-electric materials, however, require high excitation voltages and only control vibration in the micro-inch range [6].

A popular method for HCF mitigation is to implement damping methods to reduce the excessive stresses in engine components that lead to high cycle fatigue [3]. Advances in manufacturing techniques have enabled integrally bladed disk designs, which are are highly susceptible to high cycle fatigue as they are inherently low damped [7]. These advanced, high performance turbomachinery blade designs have led to higher stresses and decreased damping.

An important metric in damping performance is the quality factor, $Q$. As damping increases, $Q$ decreases. A structure that is exhibiting higher damping will have a lower $Q$ value. The typical $Q$ value of a blade structure is 1000 or greater. A $Q$ value of 100 or less is desirable to reduce vibratory stress in the blade. Using FEA, Duffy et al. found that for a $1.8 \mathrm{in.} \times 0.75 \mathrm{in} . \mathrm{x} 0.010 \mathrm{in}$. beam, with small 0.005 in. SMA patches on both sides, if the $Q$ value of the SMA structure was 14 , the $Q$ value of the composite structure would be 100 . During material testing 
of $\mathrm{Ni}_{50} \mathrm{Ti}_{35} \mathrm{Hf}_{15}$, Duffy et al. found minimum quality factors of less than 5, but these factors corresponded to the low input frequency of $0.1 \mathrm{~Hz}$. See Figure 1 where the loss factor $\eta$ represents the ratio of damping energy to strain energy and is equal to the reciprocal of $Q$. No damping peak was found in the $\mathrm{Ni}_{50} \mathrm{Ti}_{35} \mathrm{Hf}_{15}$ material at $100 \mathrm{~Hz}$ [3].

Commercially shape memory alloy Nickel Titanium (NiTi, Nitinol) has been used in many applications including ocean engineering [8], civil engineering [9] and a host of mechanical engineering areas. NiTi has demonstrated damping an order of magnitude higher than ordinary metals [10]. Piedboeuf et al. found a minimum quality factor at $0.1 \mathrm{~Hz}$ in their own study of NiTi wires [11]. Ikegami investigated active control with NiTi wires and piezo-electric ceramic actuators [12].

Damping characteristics in NiTi alloy are influenced by the composition and the thermo-mechanical processing [3]. The transformation temperature can be tailored through formulation, especially for high temperature applications $[13,14] ; \mathrm{Ni}_{19.5} \mathrm{Ti}_{50.5} \mathrm{Pd}_{25} \mathrm{Pt}_{5}$ has a transformation temperature near $300^{\circ} \mathrm{C}$, which is conductive for turbomachinery applications [15]. The transformation temperature of NiTi can be changed by increasing or decreasing the atomic percentage of $\mathrm{Ni}$ in the material. NiTi with $50 \% \mathrm{Ni}$ exhibits a transformation temperature of $120^{\circ} \mathrm{C}$, this temperature decreases with increasing nickel content. NiTi 55 has shown superior corrosion resistance when compared to stainless steel in salty environments. It also can be hot formed into various shapes and does not require cold working [16].

Shape memory alloys allow for large amounts of recoverable strain which coincide with work densities that can be ten times higher than traditional approaches. The shape memory effect allows for the use of shape memory alloys in applications where work per unit weight must be maximized. The density of NiTi is $6.45 \mathrm{~g} / \mathrm{cm}^{3}$, slightly higher than the $4.43-4.66 \mathrm{~g} / \mathrm{cm}^{3}$ density of current titanium alloys used in aero engine components [17]. Shape memory alloys can be used for multiple activations. If the material is returned to its original state, it can be deformed, reheated, and recovered repeatedly [18].

The hysteresis an SMA undergoes represents the mechanical energy it can dissipate. Damping can occur in shape memory alloys during the solid-solid phase transition, during which energy can be dissipated. Duffy et al. found that SMAs exhibit damping during their phase transformation. The amount of damping observed depends upon the steady state stress level, alternating stress level, frequency, and temperature.

SMAs have been used in many fixed-wing aircraft, rotorcraft, and spacecraft applications. SMA's have been used to create hingeless ailerons and variable geometry airfoils, as well as variable inlets and exhaust chevrons. They have been used in satellite low-shock release mechanisms because of their slow activation. Most applications are designed through careful, specific experimentation. Civil structural component designers have been interested in SMAs because of their ability to dissipate energy through hysteresis [16]. These applications illustrate that SMAs have become a viable engineering material for aerospace and vibratory components.

Shape memory alloys exhibit a change in elastic modulus when they undergo the phase transition from martensite to Austenite. This modulus change creates variable stiffness in components utilizing shape memory material. The stiffness change from the martensite to Austenite phase makes SMAs a tool to isolate vibrations. It has been shown that the variable stiffness property inherent to shape memory alloys has the capability of shifting the natural frequencies of components made with this material. Along with a frequency shift, variable stiffness has been shown to reduce component amplitudes and create a change in the mode shape vector $[1,19,20]$. Preliminary damping quantification suggested NiTI effectiveness as a HCF mitigation technique [20].

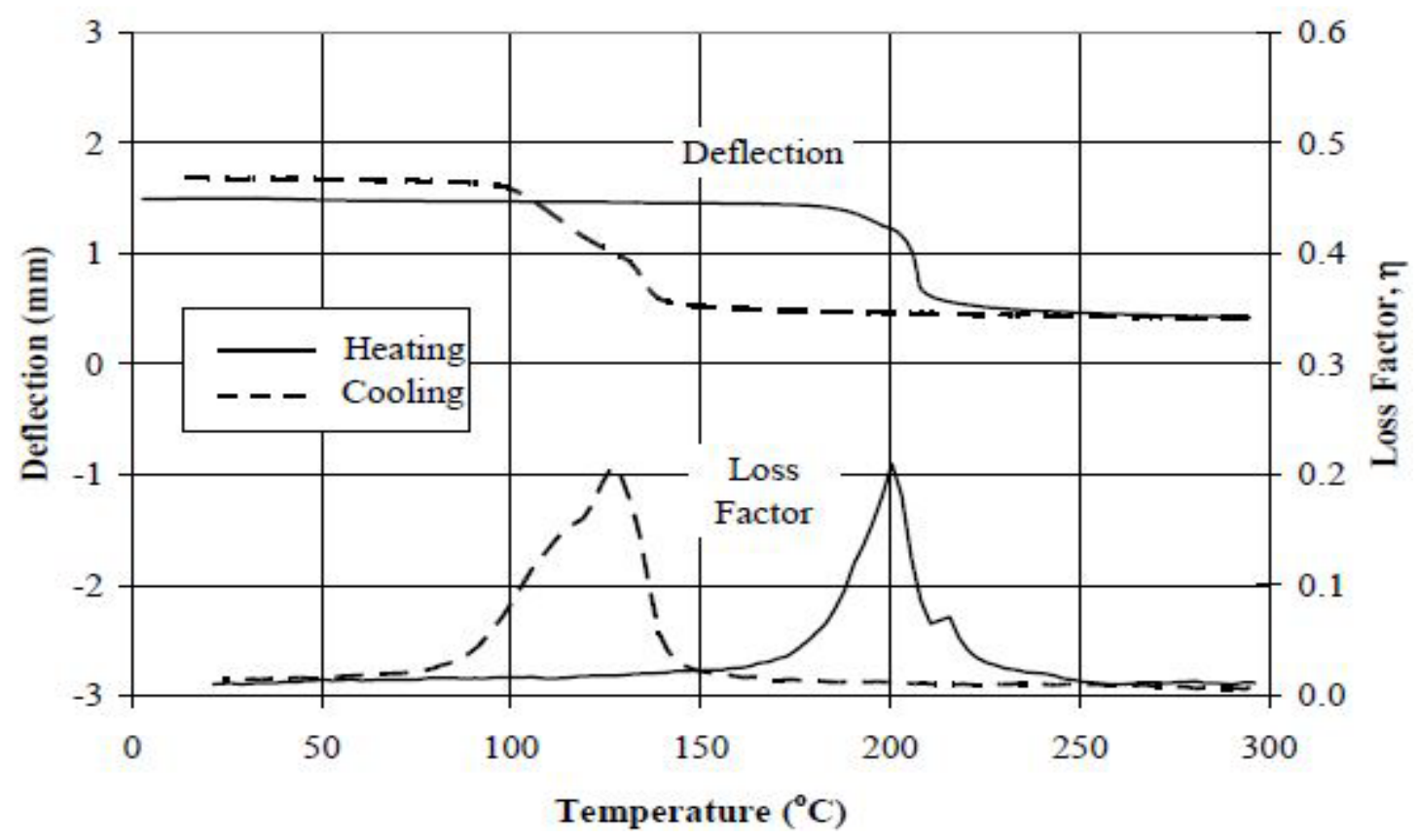

Figure 1. Cast Ni50Ti35Hf15 static test deflection and dynamic test loss factor temperature. Loss factor at $0.1 \mathrm{~Hz}$, 35×10-6 strain amplitude [3] 


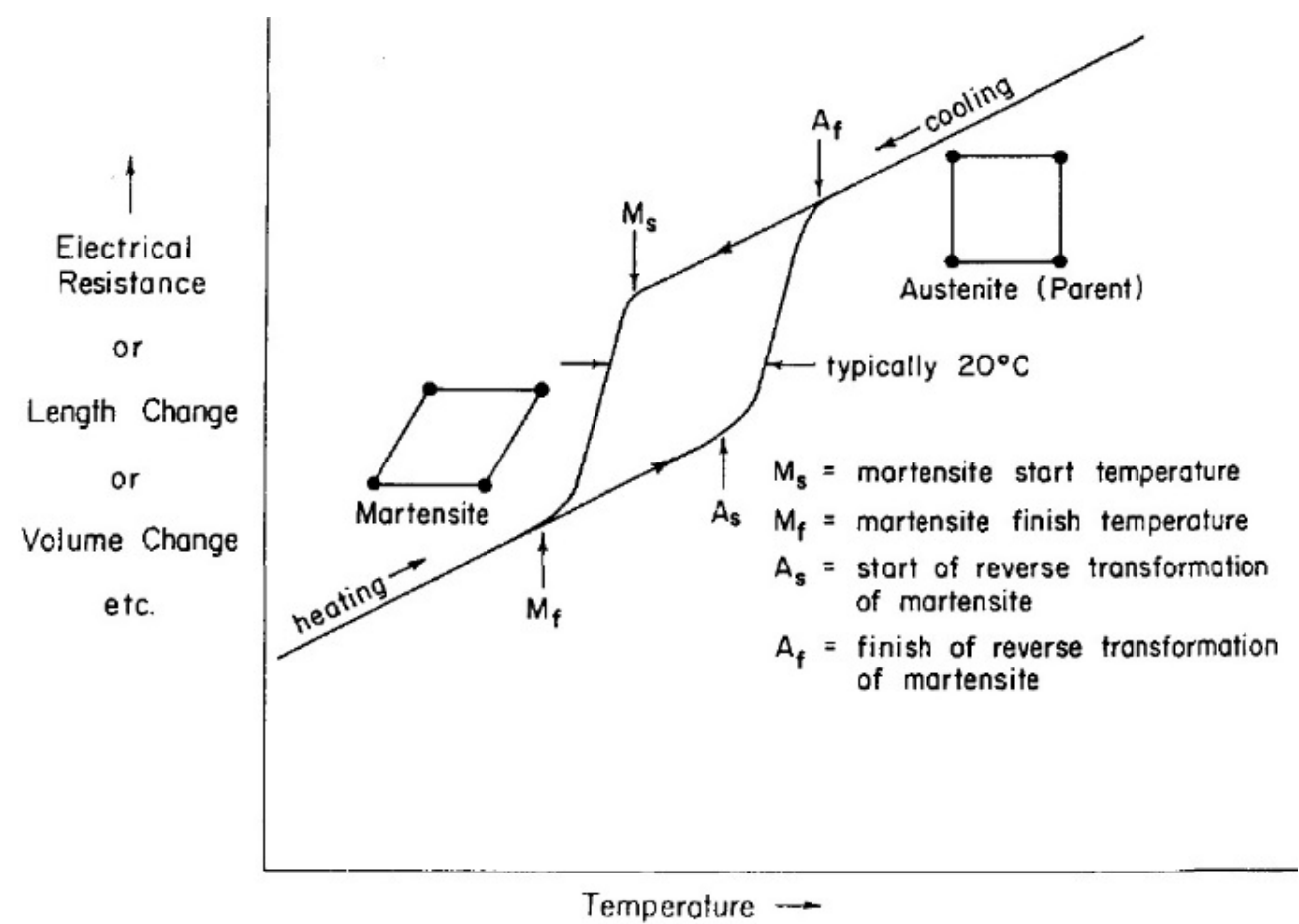

Figure 2. Hypothetical plot of property change vs. temperature for the martensite to austenite phase transformation in a shape memory alloy [18]

\section{Technical Approach}

The shape memory effect is present in several materials. One such material of interferes is the nickel - titanium based Nitinol [18]. The materials has the unique property of returning to shape if this material is deformed while in the martensitic phase and then subsequently heated above $A_{f}$, the Austenite finish temperature. This behavior is due to a solid-solid phase change in the material from martensite to austenite and is often characterized as pseudo-plasticity. An illustration of the hysteretic loop shape memory alloys undergo during their solid-solid phase changes is shown in Figure 2 [18]. The phase transformation is evident in the non-linear property response. As the sample is heated, a nonlinear property change is evident at the austenite start temperature $\left(A_{s}\right)$ and continues until it reaches the austenite finish temperature $\left(\mathrm{A}_{\mathrm{f}}\right)$. Further heating is shown as a linear response. Upon cooling, the non-linear property change is evident at the martensite start temperature $\left(M_{s}\right)$ and continues until it reaches the martensite finish temperature $\left(\mathrm{M}_{\mathrm{f}}\right)$. These changes are extended to include changes in elastic modulus. SMAs can absorb and dissipate energy through their hysteretic shape change when they are cyclically loaded. The properties of SMAs have made them suitable for many sensing and actuation applications [16]. The introduction of the shape memory effect, whereby the shape memory alloy material acts as both a damper and a means of variable stiffness is a novel technique for HCF mitigation. Electromagnetic actuators have is also a methods for controlling vibration amplitude [21].

Shape memory alloys exhibit a change in elastic modulus when they undergo the phase transition from martensite to austenite. This modulus change creates variable stiffness in components utilizing shape memory material. In has been shown that the variable stiffness property inherent to shape memory alloys has the repeatable capability of shifting the natural frequencies of components made with this material. Repeated tests showed consistent change in modal frequency during shape memory alloy phase change. Highlighting this capability, a modal frequency change of $5.95 \%$ to $6.53 \%$ was realized during the cooling of a composite sample over modes 2-5 [19]. Along with a frequency shift, variable stiffness has been shown to reduce component amplitudes and create a change in the mode shape vector [19].

The hysteresis an SMA undergoes represents the mechanical energy it can dissipate. The target application of SMA damping in turbomachinery would be its use on a compressor blade. Here frequencies can range from 100 to $10,000+\mathrm{Hz}$. The "quality factor", $Q$, is a dimensionless parameter that describes the damping of a structure. The lower the $Q$ value, the higher the damping is in the structure. Damping is calculated by using the "half-power bandwidth" method by measuring the bandwidth of the fast Fourier transform (FFT) curve below the resonant peak [4]. The damping ratio, $\zeta$, is found by:

$$
\zeta=\frac{\Delta \omega}{2 \omega_{r}} .
$$

Here $\Delta \omega$ represents the bandwidth defined as $\left(\omega_{2}-\omega_{1}\right)$ and $\omega_{r}$ is the resonant frequency. The quality factor is inversely proportional to the damping ratio and is calculated as:

$$
Q=\frac{1}{2 \zeta}
$$

The objective of the research presented was to map and quantify the effectiveness of the shape memory alloy Nitinol in creating active damping and stiffness in a beam. Through a series of experimental analyses the effects were quantified. These experiments had two focuses: (1) map the modal shape by quantifying change in node location 
due to temperature change, and the third focus was to characterize the materials response to changing temperature while forced vibration was held at several different constant frequencies, and (2) investigate changes in damping during the solid-solid phase change of the shape memory material.

\section{Experimental Approach}

A modal analysis was performed on a cantilever beam in a fixed-free condition to simulate a simple, vibrating turbomachinery component. The performance of the beam was mapped to provide guidance in future design and optimization. It was expected that optimally designed components will be of low weight and low power consumption, and thus attractive for future use in flight applications. High temperature SMA formulations exist, reaching activation temperatures around $300^{\circ} \mathrm{C}[13,14,15]$; utilization of the engine operating temperature may be a viable source of actuation.

\subsection{Test Specimen Design}

This research effort leverages an extremely large experimental and theoretical base. Test specimens were created and consistent with those used to quantify the eigenvalue, eigenvectors, and amplitude changes in recent studies $[1,19,20]$. The test specimens consisted of a topical treatment adhered to an aluminum substrate. The specimens were designed to have a free length of 8 in. when clamped to a dynamic shaker. The overall dimensions of the aluminum specimens was 11 in. $x 1$ in. $x 0.125$ in.

Two topical treatment were fabricated. The dimensions of the topical treatment are illustrated in Figure 3; further differentiated in length as "full-span" and "half-span". The experiment test specimens consisting of one "half-span" and one "full-span" were created from shape memory alloy sheet material NiTi. The experimental control was fabricated from aluminum topical sheet in only the "full sample" configuration. The shape memory topical treatment consisted of $\mathrm{Ni}_{55} \mathrm{Ti}_{45}$ and was received as factory annealed flat. The selected SMA configurations were cut out of the 0.008 in thick shape memory sheet material by a waterjet. The treatments were not subjected to any additional heat training. Certificates of material data accompanying the shape memory alloy sheet material listed the modulus of the material as $28-41 \mathrm{GPa}$ for the martensite phase and $83 \mathrm{GPa}$ for the austenite phase. The austenite finish temperature of the material was listed as $113^{\circ} \mathrm{C}$. The control topical treatment consisted of Aluminum 1145-H19 and was 0.008" thick.

The substrate of the test specimens consisted of a beam, machined from 0.125 in. aluminum alloy 6061 sheet material. Again, the specimens were designed to have a free length of $8 \mathrm{in}$. when clamped and an overall dimensions of 11 in. $x 1$ in. $x 0.125$ in.

The SMA treatments were adhered to one side of the aluminum alloy beams using Permabond 920 high temperature cyanoacrylate. The treatments were designed to be fully adhered to the 8 in. free length of the beam while also being applied to the section of the beam that ran under the clamp so that the SMA could be attached to leads on the opposite side of the clamp. The composite samples underwent a full curing process to leverage the high temperature properties of Permabond 920. During this process the samples were brought to $150^{\circ} \mathrm{C}$ for two hours in an oven. Prior to the adhesion process, the aluminum beam specimens were anodized to electrically isolate them from the SMA treatments. Continuity testing of the anodized aluminum substrates and SMA treatments in the adhered condition ensured the electrical isolation between the components. The experimental control consisted of a thin aluminum foil, adhered to the same anodized aluminum substrate. Composite samples can be seen in Figure 4.

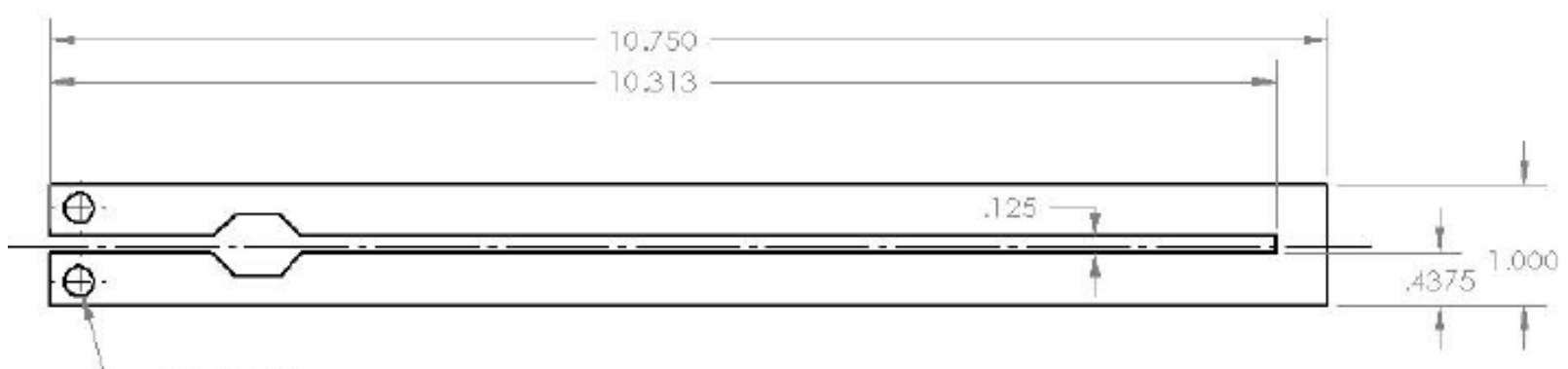

(a) SMA treatment for full-span sample

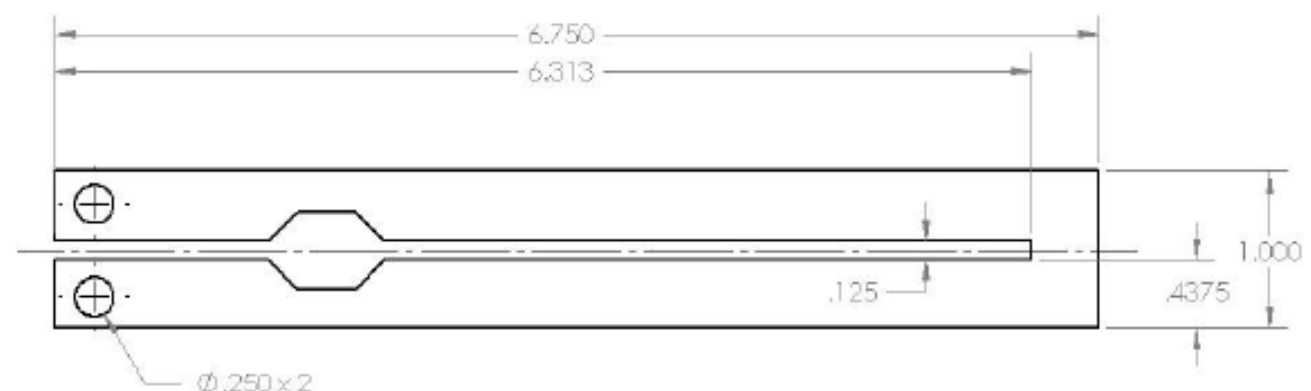

(b) SMA treatment for half-span sample

Figure 3. Dimensions of shape memory topical treatment: (a) full-span length and (b) half-span length 


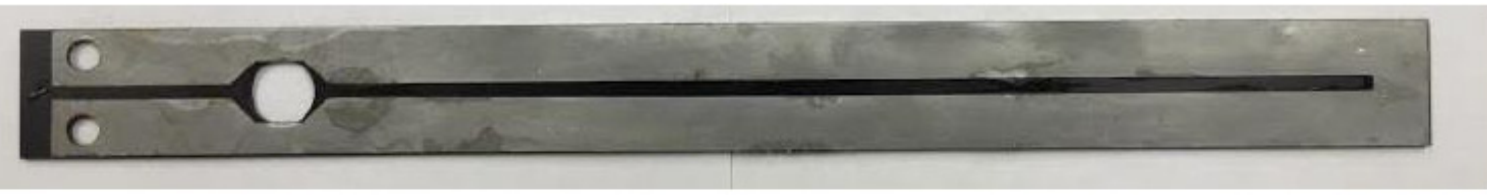

(a) Full-span SMA treatment adhered to aluminum substrate.

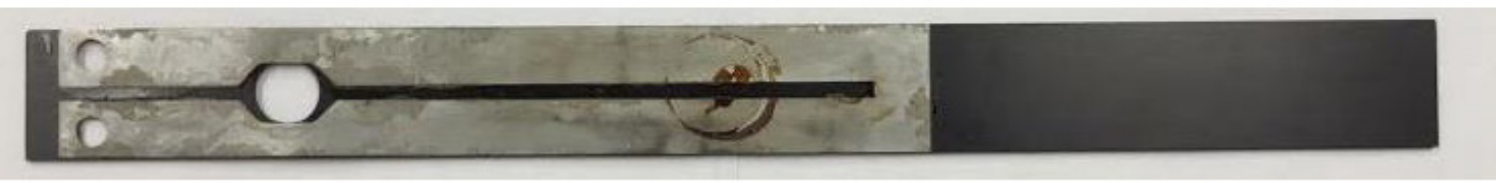

(b) Half-span SMA treatment adhered to aluminum substrate.

Figure 4. Composite experimental samples with SMA treatment: (a) full-span and (b) half-span

\subsection{Experimental Method}

Experimental methods included the use of a single point scans of the beam tip using a Polytec laser vibrometer. The shift in frequency and damping was quantified at multiple temperature points, as the SMA patch was brought from room temperature, past activation temperature, and then back down to room temperature. These scans were used to illustrate any change in the beam's damping and stiffness characteristics as a result of phase change.

In order to control temperature, Joule heating was utilized as the catalyst in obtaining the original shape from the deformed SMA. This method allowed for a low power mechanism to return the SMA patch to its original state and orientation. Employing this method allowed for a low power mechanism to return the SMA patch to its original state and orientation. Low weight is inherent to the use of SMAs because they exhibit large amounts of recoverable strain with direct actuation [18]. It is expected that optimally designed components will be of low weight and low power consumption, and thus attractive for future use in flight applications. Alternatively, SMA formulations exist with high temperature transformation temperatures $[13,14,15]$; utilization of the engine operating temperature may be a viable source of actuation.

The surface temperature of the SMA patches was controlled with the use of a DC power supply. Leads were attached to either end of the SMA loop on the opposite side of the dynamic shaker clamp so as to not interfere with vibratory testing. Temperature readings of the SMA patches were taken using an infrared temperature sensor and the information was fed to a data acquisition system. A LabVIEW controller used the feedback data and a specified target temperature to control the SMA surface temperature by varying the current that was flowing through the patch.

Vibratory excitation was provided by a 6000 pound dynamic shaker apparatus. Instrumentation included an accelerometer attached to the top of the clamp, IR temperature sensor, and a Polytec single point laser vibrometer. The samples were clamped on one end by the dynamic shaker, the other end remainted free. In order to isolate the beam for Joule heating, ceramic inserts were fabricated from the shaker head made from Very High Temperature Machinable Glass-Mica Ceramic with a maximum operating temperature of $1100^{\circ} \mathrm{F}$. Inserts were used on either side of the beam, sandwiching it between the shaker's steel clamp faces. A Teflon washer was used to further isolate the clamp's center bolt from the SMA patch.
The vibration single was acquired with a Polytec OFV-505 single point laser vibrometer, connected to a Polytec OFV-5000 controller. The laser was mounted directly above the shaker, sample, and clamp and the laser beam was manually aligned to the tip of the beam. The controller autofocus was used to focus the laser. VibrationVIEW software was used to control shaker input, acquire laser data, perform all necessary FFTs, and output results. The sine test module was used in both sweep and fixed-frequency scans. The software controlled the shaker to an acceleration equal to $0.1 \mathrm{G}$. In damping studies, this software also used FFT output to perform a calculation for the damping "quality factor", $Q$ using the half-power bandwidth method. The software also output the peak frequency and amplitude of the frequency range studied.

\subsubsection{Frequency Performance Mapping}

The performance of the test specimens was mapped at a constant excitation frequency. These simulated an engine test running at cruise. A series of four tests were completed, two on the half sample and two on the full sample. For each test the sample remained clamped to the 6000 pound shaker while a series of frequency dwells were performed. During each dwell, the joule heating controller was used to heat the sample and then allow it to cool. These frequency dwells were repeated at several different frequencies to quantify the response of the sample under different circumstances.

The second bending mode was excited for both half-span and full-span samples, occurring around $200 \mathrm{~Hz}$ in both. Accordingly, dwell frequencies around $200 \mathrm{~Hz}$ were used for testing. Multiple single point scans were completed to quantify damping effects. A reduction of the laser vibration signaled generated FFT data for each temperature investigated using VibrationView. The software was used to map the time domain data to the frequency domain and takes the ratio of the response to the input data to calculate the Frequency Response Function (FRF). The FRF was used to identify modal frequencies.

\subsubsection{Damping Quantification}

Damping was quantify with a 6000 pound shaker with the ceramic inserts. VibrationVIEW software was used to generate a sine sweep in the shaker that captured second bending mode in the samples. The samples were heated and allowed to cool through use of the Joule heater. Multiple single point scans were completed to quantify damping effects. In order to capture the quality factor, Q, 
with good resolution, the best practice of sweeping a frequency range of two times the modal peak bandwidth was employed. Several scans were taken at each set temperature value to ensure the sample was being swept in this way. The sweeps were completed over an approximately $4 \mathrm{~Hz}$ frequency range.

\section{Results and Discussion}

The frequency and damping performance was mapped for $\mathrm{Ni}_{55} \mathrm{Ti}_{45}$-aluminum composite beam. Both frequency shift mapping and damping experimentation scans were taken at multiple temperature points, as the SMA patch was brought from room temperature, past activation temperature, and then back down to room temperature. These scans were used to illustrate any change in the beam's damping and stiffness characteristics as a result of phase change.

\subsection{Frequency Mapping}

A key performance metric for this technique is a mapping of the frequency-temperature domain. In order to reduce the data, the vibration signal for all of the frequency shift mapping scans were compiled and filtered for noise with the peak-to-peak velocities converted to amplitudes. Representative dwell data for the full sample for two frequencies, $199 \mathrm{~Hz}$ and $201 \mathrm{~Hz}$, are shown in Figure 5 and Figure 6 . It is evident that the change in response between the two dwell frequencies is caused by the phase change inherent in $\mathrm{Ni}_{55} \mathrm{Ti}_{45}$. Both the temperature increasing curve and the temperature decreasing curve exhibit two peaks at relatively the same amplitude. These two modal peaks are due to the changing stiffness in the material. At $201 \mathrm{~Hz}$, the difference between the two peaks is approximately $73.5^{\circ} \mathrm{C}$ for increasing temperature and $38.5^{\circ} \mathrm{C}$ for decreasing temperature, at $199 \mathrm{~Hz}$, these values are $23.6^{\circ} \mathrm{C}$ and $11.1^{\circ} \mathrm{C}$ respectively. The response's amplitude is dependent on both temperature and input frequency.

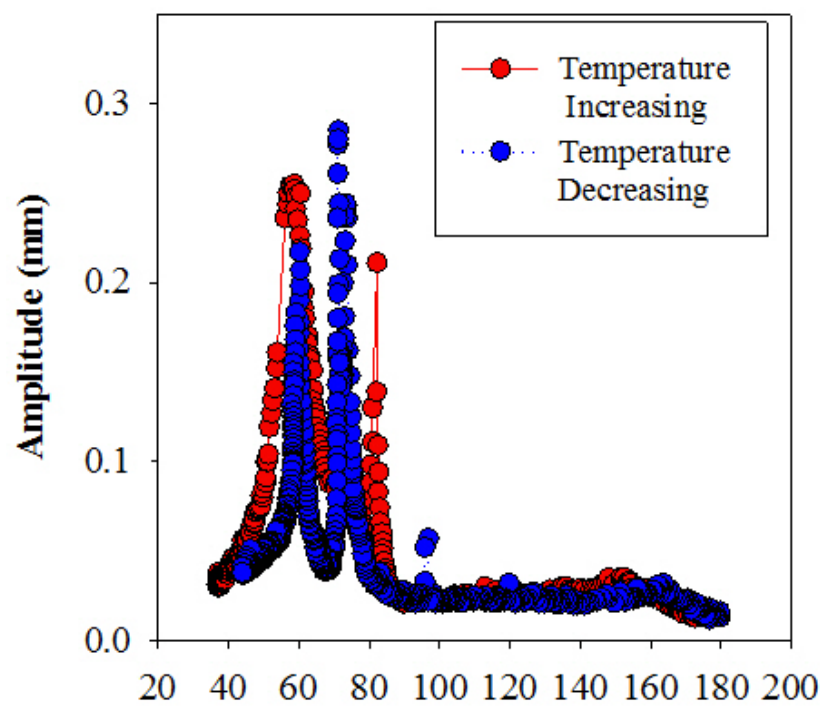

Temper ature $\left({ }^{\circ} \mathrm{C}\right)$

Figure 5. Example of Temperature vs. Tip Amplitude, Full Sample, 199 $\mathrm{Hz}$ dwell



Figure 6. Example of Temperature vs. Tip Amplitude, Full Sample, 201 $\mathrm{Hz}$ dwell

Performance maps were created to better understand the temperature-frequency relationship. Performance were created utilizing the MATLAB code gridfit to map the data to a surface plot [22]. Here, a triangular interpolation with gradient smoothing regularization is utilized to convert the three dimensional point data into a meaningful surface. Representative amplitude maps for the temperature and frequencies tested are shown in Figure 7 for the fullspan test specimen and Figure 8 for the half-span test specimen. Note that scans 2 for the half sample used data from five dwells, while the other scans used data from 7 dwells.

The nonlinear dynamic response of the test sample is evident in the temperature vs. modal frequency vs. amplitude maps for second bending mode. As a design tool, these maps graphically depict how tip amplitude changes with changing temperature. They can be utilized by engineers to design a component to actively avoid areas of high strain. The maps also provide guidance for active controller, whereas the frequency can be controlled through heat addition or removal. An example of this use in the half sample (Figure 8) may occur if a component was operating and the excitation frequency was increasing to $202 \mathrm{~Hz}$ at $60^{\circ} \mathrm{C}$. Actuating the sample and changing its temperature to $100^{\circ} \mathrm{C}$ would decrease the tip displacement.

\subsection{Damping}

Damping was quantify using the quality factor metric. The quality factor with a frequency response superimposed is shown in Figure 9. Both the heating and cooling cycles are shown, indicted minimal hysteresis in quality factor with temperature. Results suggested that the quality factor does not changed drastically during phase transition. The quality factor response was somewhat constant at a value around 200 to 250 with a notable drop to a value around 100 at the highest temperature scans in both samples. It is suggested that drop at high temperatures may also be influenced by softening effects in the beam at these temperatures. While Duffy et. al had 
predicted an increase in damping during phase change, during the experimental investigation, Q stayed fairly constant. Duffy et. al's prediction was for a free piece of shape memory alloy material while this damping investigation was completed on a composite NiTi-aluminum alloy structure. This would account for less than optimal damping realization during the phase transition as the aluminum substrates was dominate.

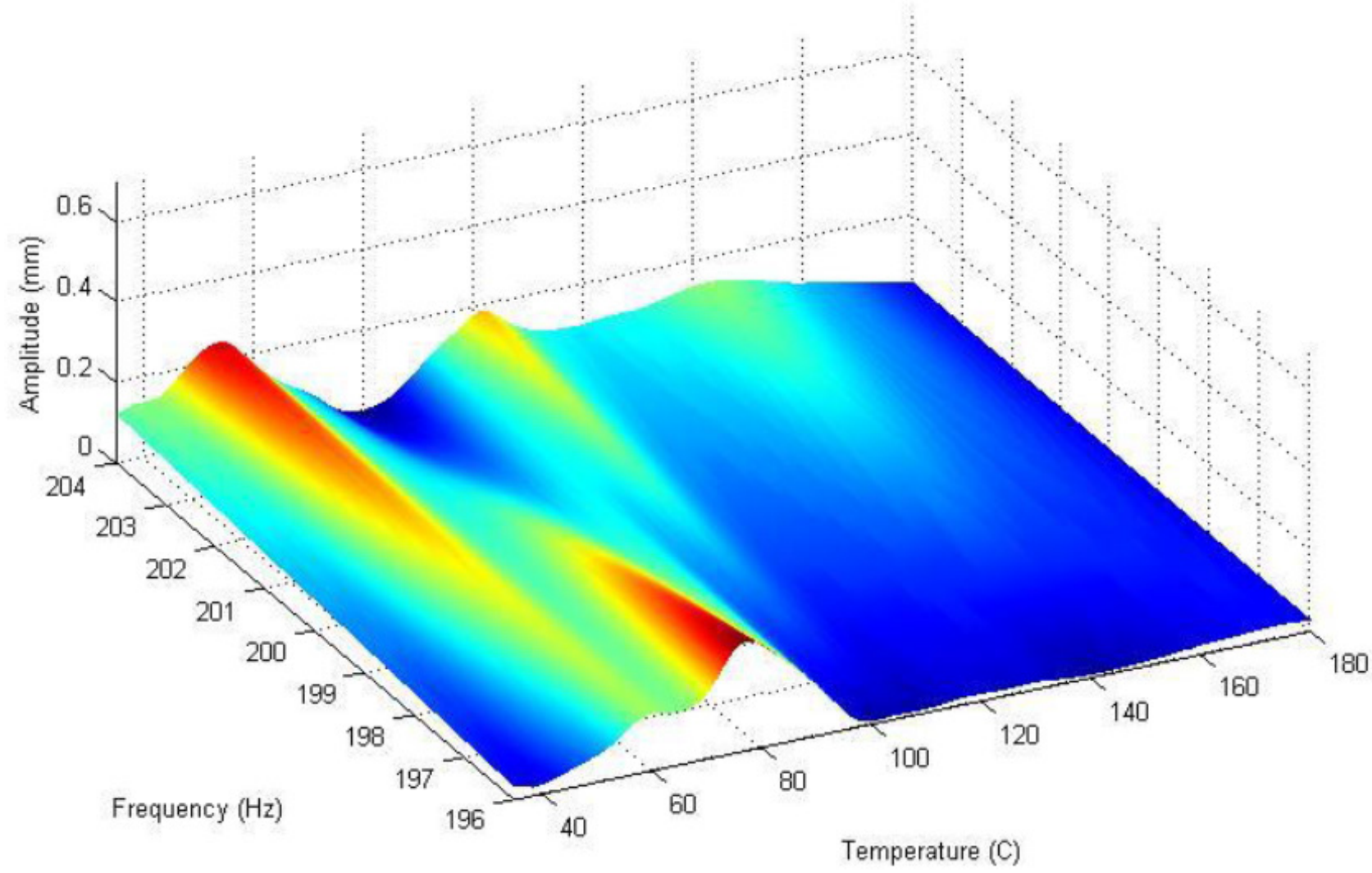

(a) Increasing temperature

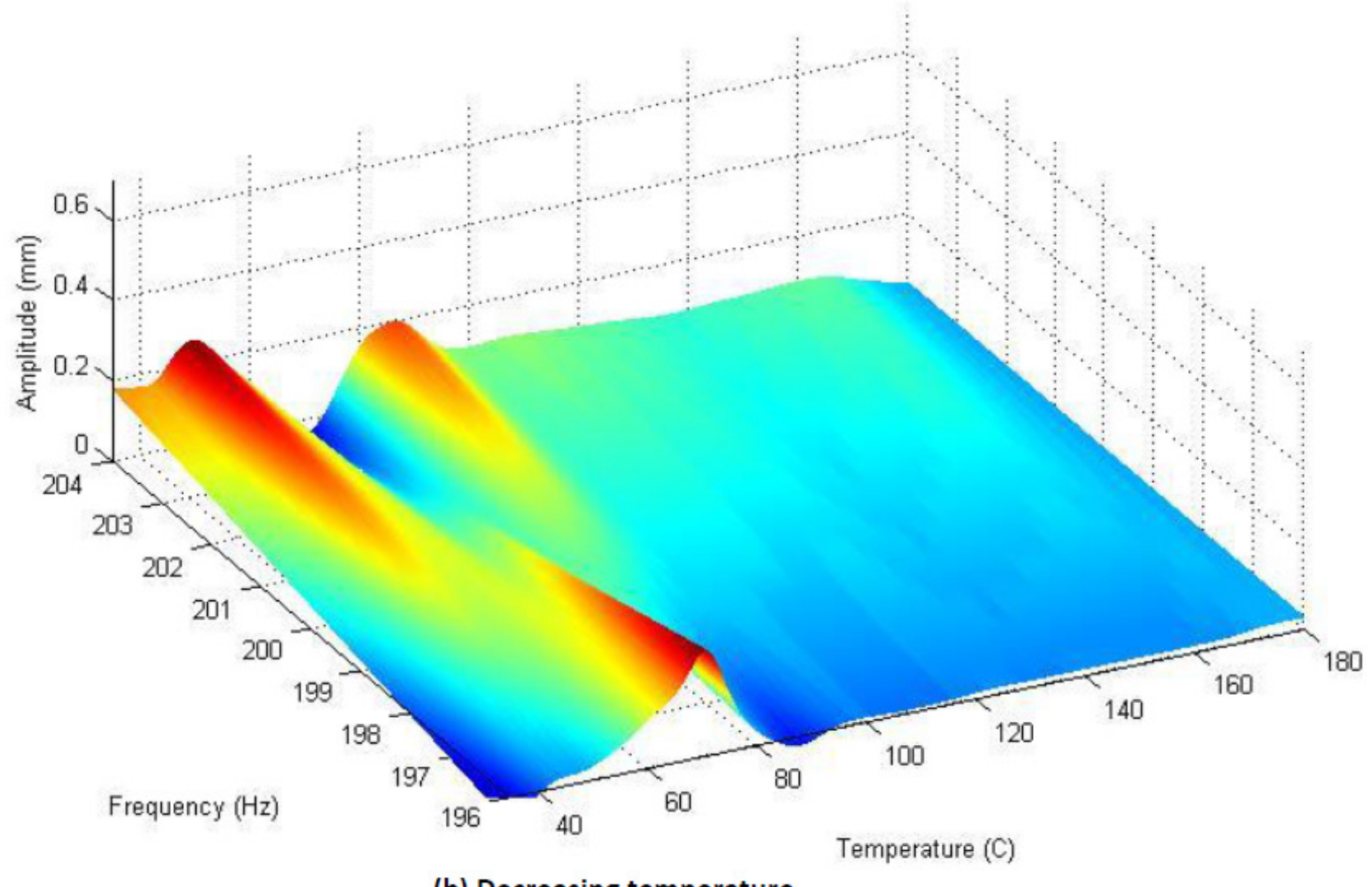

(b) Decreasing temperature

Figure 7. Performance map of full-span sample: (a) increasing temperature and (b) decreasing temperature 


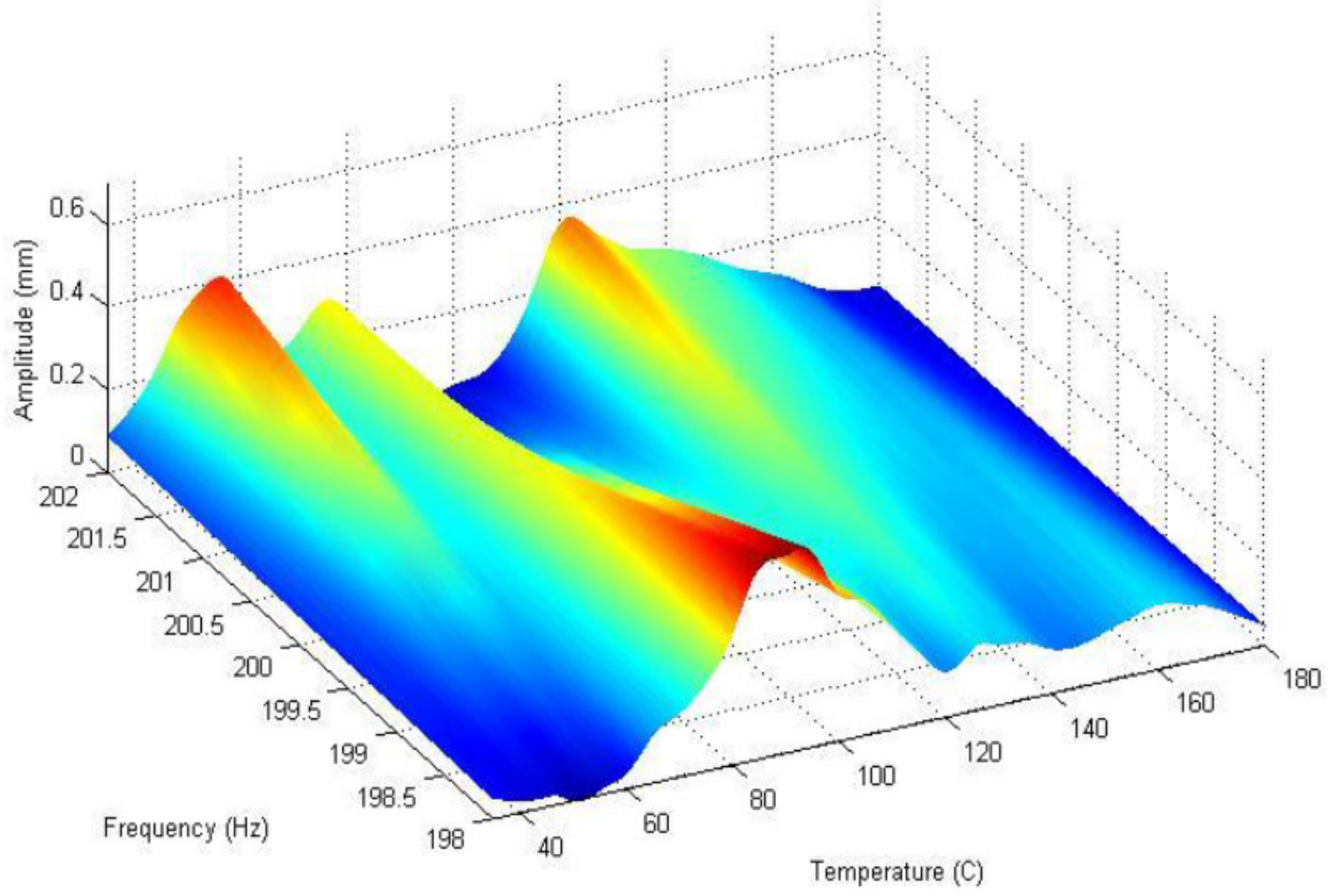

(a) Increasing temperature

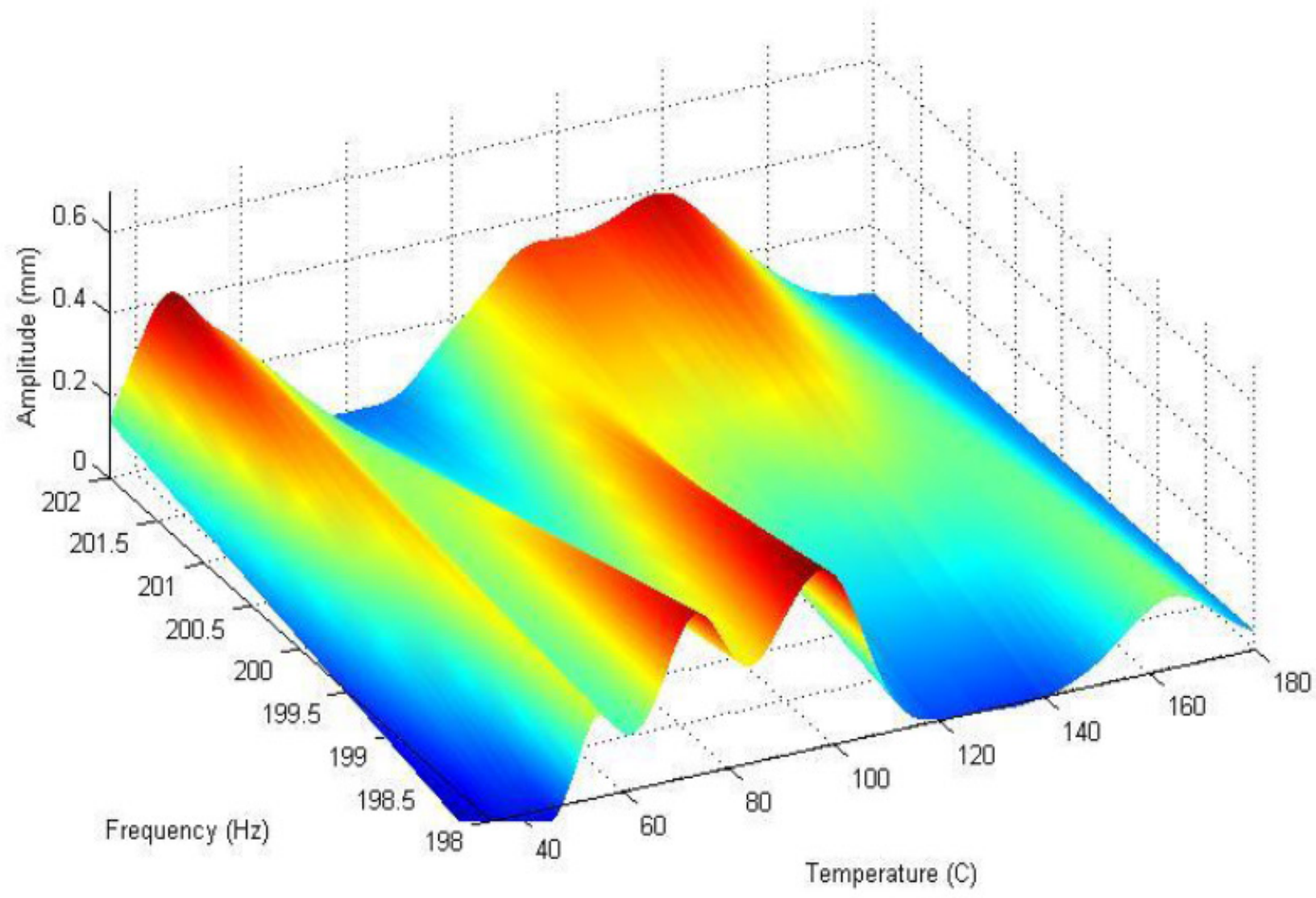

(b) Decreasing temperature

Figure 8. Frequency performance map of half-span sample: (a) increasing temperature and (b) decreasing temperature 


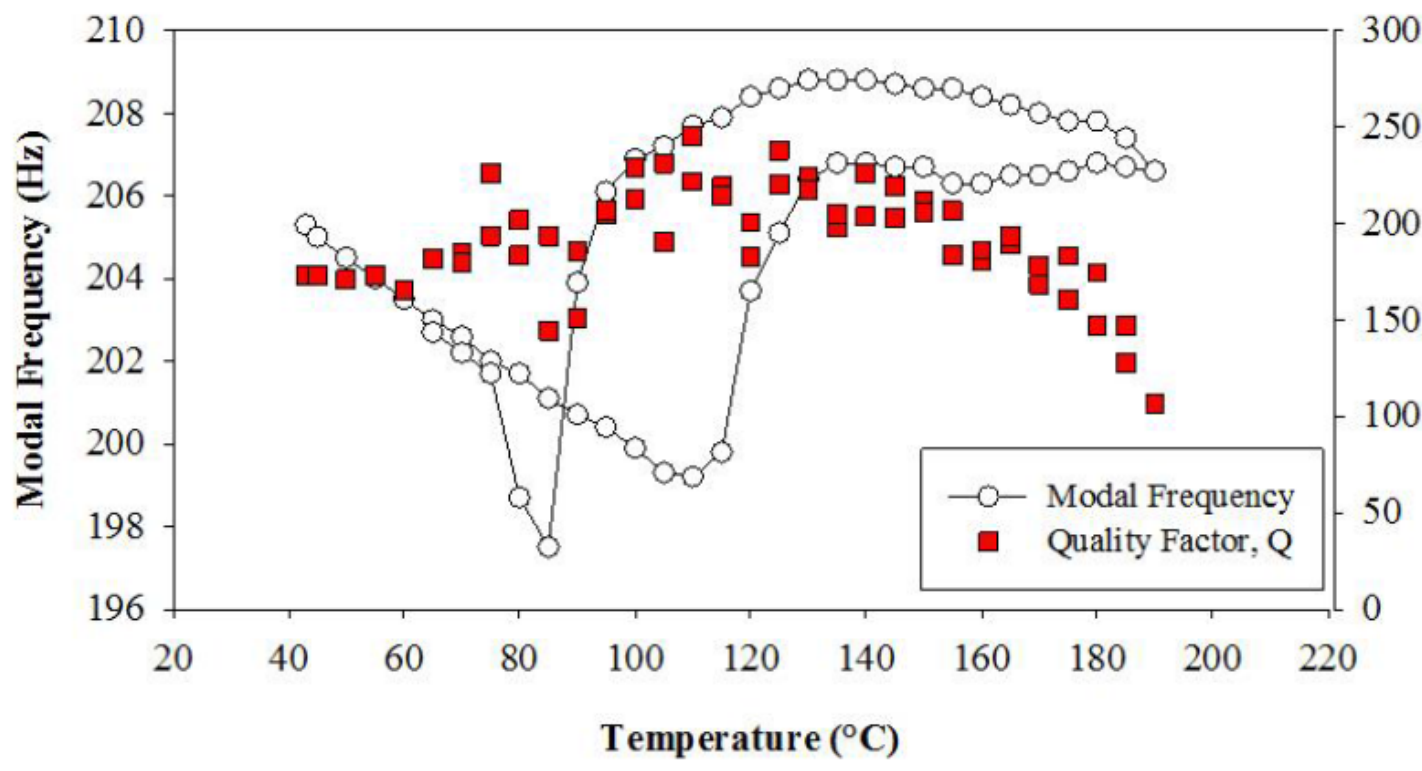

(a) Full-span damping performance

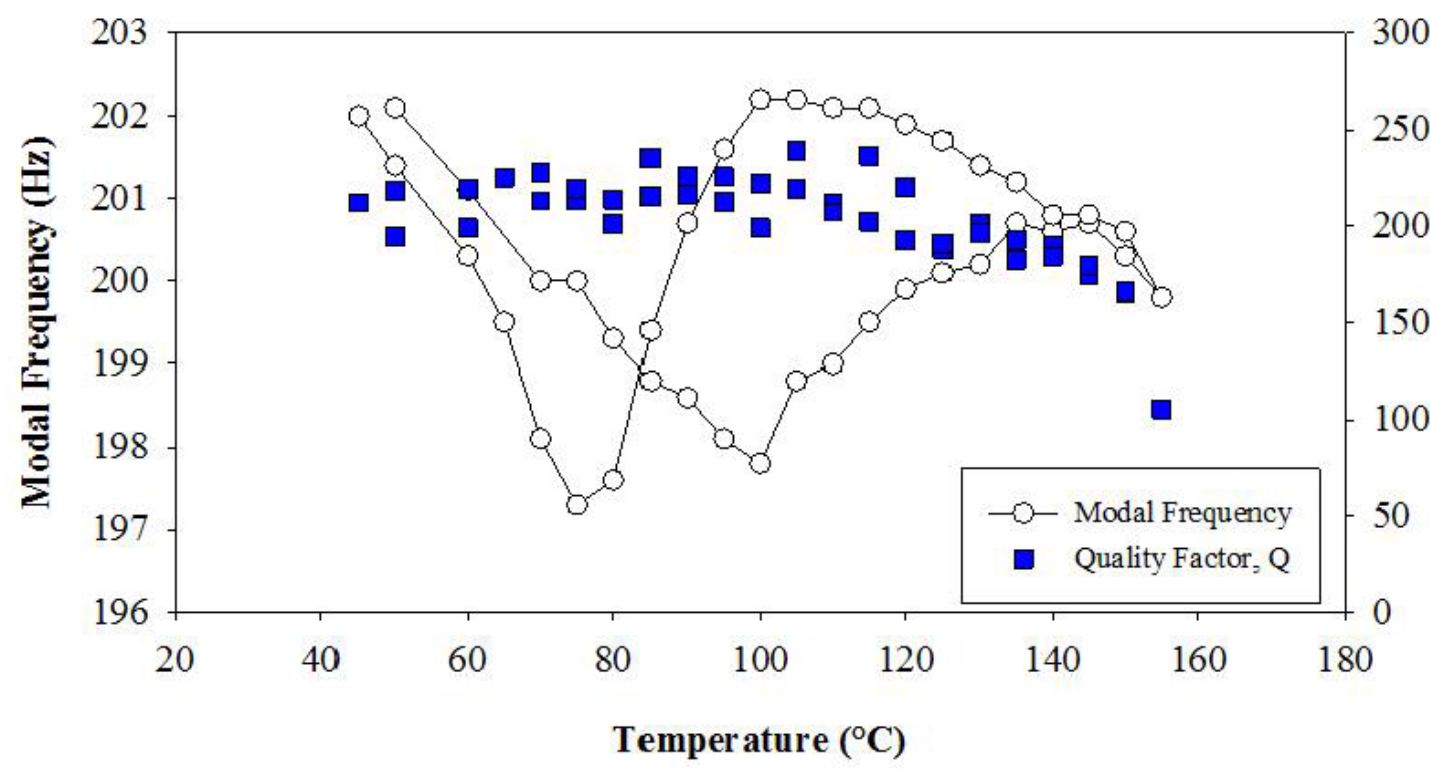

(b) Full-span damping performance

Figure 9. Damping performance map of half-span sample: (a) increasing temperature and (b) decreasing temperature

\section{Conclusions}

Novel methods and materials are important for effective high cycle fatigue mitigation. State-of-the art compressor blades and stators have increased turbomachinery performance, all while increasing the vibratory stresses (e.g., integrally bladed disks). The research presented focused on the feasibility of the use of the shape memory effect of shape memory alloys as active actuators to mitigate stresses and dampen blades. The performance of two composite samples in a simple geometry, designed to leverage the strengths of the "smart materials."

Damping investigations included charactering the quality factor for the hysteretic cycle of composite beam. Results suggested an increased damping with increasing temperature. A peak in quality factor during phase transformation was not realized.

Performance charts of temperature, modal frequency and beam tip amplitudes where established for a range of working temperatures. Results illustrated the design capabilities for a composite using shape memory alloys. The investigation qualified the change in response that can be realized with the use of a small SMA actuator. In summary, NiTi's unique capability in creating variable stiffness in a blade as well as its effect on damping characteristics has been mapped.

\section{Acknowledgements}

The authors appreciate the contributions of Dr. Jeffrey Brown, Dr. Tommy George, and the Turbine Engine Fatigue Facility (TEFF) at The Air Force Research Laboratory. This work was supported by the Dayton Area Graduate Studies Institute and the Air Force Research Laboratory Ohio Student-Faculty Research Program under agreement RQ6-UA-14-5-AFRL. 


\section{References}

[1] Garafolo, N. G., and Collard, R., 2017. "Active stiffness method for high cycle fatigue mitigation using topical thin foil shape memory alloy". Journal of Mechanical Design and Vibration, 5(1), pp. 11-20.

[2] Cowles, B. A., 1996. "High cycle fatigue in aircraft gas turbines an industry perspective". International Journal of Fracture, 80, pp. 147-163.

[3] Duffy, K., Padula, S. A., and Scheiman, D., 2008. "Damping of high temperature shape memory alloys". In The 15th International Symposium on: Smart Structures and Materials \& Nondestructive Evaluation and Health Monitoring.

[4] Blackwell, C., Palazotto, A., George, T. J., and Cross, C. J., 2007. "The evaluation of the damping characteristics of a hard coating on titanium". Shock and Vibration, 14(1), pp. 37-51.

[5] Easterday, O., Palazotto, A., Branam, R., Baker, W., and George, T., 2011. "Experimental characterization of damping properties of coatings at elevated temperatures using a free-free beam based apparatus". In 52nd AIAA/ASME/ASCE/AHS/ASC Structures, Structural Dynamics and Materials Conference, American Institute of Aeronautics and Astronautics.

[6] Baz, A., Imam, K., and McCoy, J., 1990. "Active vibration control of flexible beams using shape memory alloys". Journal of Sound and Vibration, 140, pp. 437-456.

[7] Bhaumik, S., Bhakaran, T. A., Rangaraju, R., Venkataswamy, M., Parameswara, M. A., and Krishnan, R. V., 2002. "Failure of turbine rotor blisk of an aircraft engine". Engineering Failure Analysis, 9(3).

[8] Buehler, W. J., and Wang, F. E., 1968. "A summary of recent research on the nitinol alloys and their potential application in ocean engineering". Ocean Engineering, 1(1), pp. 105-120.

[9] McGavin, G. L., and Guerin, G., 2002. "Real-time seismic damping and frequency control of steel structures using nitinol wire". Proc. SPIE, 4696, pp. 176-185.

[10] Humbeeck, J. V., 2003. "Damping capacity of thermoeleastic martensite in shape memory alloys". Journal of Alloys and Compounds, 355(1), pp. 58-64.

[11] Piedboeuf, M. C., and Gauvin, R., 1998. "Damping behavior of shape memory alloys: strain amplitude, frequency and temperature effects". Journal of Sound and Vibration, 214, pp. 885-901.
[12] Ikegami, R., Wilson, D. G., Anderson, J. R., and Julien, G. J., 1990. "Active vibration control using nitinol and piezoelectric ceramics". Journal of Intelligent Material Systems and Structures, I(2), pp. 189-206.

[13] Noebe, R., D. Gaydosh, D., S. Padula, I., Garg, A., Biles, T., and Nathal, M., 2005. "Properties and potential of two (NiPt)Ti alloys for use as high-temperature actuator materials". pp. 364-375.

[14] Noebe, R., S. Padula, I., Bigelow, G., Rios, O., Garg, A., and Lerchl, B., 2006. "Properties of Ni19.5Pd30Ti50.5 high temperature shape memory alloy in tension and compression". In Proceedings of Smart Structures and Materials 2006: Active Materials: Behavior and Mechanics, Vol. 6170.

[15] S. Padula, I., Noebe, R., Bigelow, G., Culley, G., Stevens, M., Penney, N., Gaydosh, D., Quackenbush, T., and Carpenter, B., 2007. "Development of a HTSMA-actuated surge control rod for high-temperature turbomachinery applications". In 48th AIAA/ASME/ASCE/AHS/ASC Structures, Structural Dynamics, and Materials Conference, no. AIAA 2007-2196.

[16] Lagoudas, D. C., ed., 2008. Shape Memory Alloys. Springer Science+Business Media, LLC, New York, NY.

[17] Klocke, F., Zeis, M., Klimk, A., and Veselovac, D., 2013. "Experimental research on the electrochemical machining of modern titanium- and nickel-based alloys for aero engine components". The Seventeenth CIRP Conference on Electro Physical and Chemical Machining (ISEM), 6, pp. 368-372.

[18] Wayman, C., 1993. "Shape memory alloys". MRS Bulletin

[19] Wischt, R., and Garafolo, N., 2015. "Variable stiffness technique for turbomachinery using shape memory alloys". Proceedings from the 56th AIAA/ASCE/AHS/ASC Structures, Structural, Dynamics, and Materials Conference.

[20] Wischt, R., and Garafolo, N., 2016. "The development of an active damping and stiffness technique for turbomachinery using shape memory alloys,". Proceedings from the 57th AIAA/ASCE/AHS/ASC Structures, Structural, Dynamics, and Materials Conference.

[21] Rajasekhar, M., and Srinivas, J., 2014. "Active vibration control in engine rotors using electromagnetic actuator system". Journal of Mechanical Design and Vibration, 2(1), pp. 25-30.

[22] D'Errico, J., 2010. Surface fitting using gridfit. http://www.mathworks.com/matlabcentral/fileexchange/8998. 